\title{
Limit theorems for the diameter of a random sample in the unit ball
}

\author{
Michael Mayer • Ilya Molchanov
}

Received: 5 July 2006 / Revised: 26 April 2007 /

Accepted: 14 May 2007 / Published online: 24 October 2007

(c) Springer Science + Business Media, LLC 2007

\begin{abstract}
We prove a limit theorem for the maximum interpoint distance (also called the diameter) for a sample of $n$ i.i.d. points in the unit $d$-dimensional ball for $d \geq 2$. The results are specialised for the cases when the points have spherical symmetric distributions, in particular, are uniformly distributed in the whole ball and on its boundary. Among other examples, we also give results for distributions supported by pointed sets, such as a rhombus or a family of segments.
\end{abstract}

Keywords Convex hull • Extreme value - Interpoint distance • Poisson process $\cdot$ Random diameter $\cdot$ Random polytope

AMS 2000 Subject Classification Primary-60G70;

Secondary-60D05, 60G55

\section{Introduction}

Asymptotic behaviour of random polytopes formed by taking convex hulls of samples of i.i.d. points has been thoroughly investigated in the literature, see, e.g., Gruber (1993), Schneider (1988) for surveys of classical results and (Reitzner 2005) for more recent studies. Consider a random polytope $P_{n}$ obtained as the convex hull of $n$ i.i.d. points $\xi_{1}, \ldots, \xi_{n}$ sampled from the Euclidean space $\mathbb{R}^{d}$.

M. Mayer · I. Molchanov ( $\varangle)$

Department of Mathematical Statistics and Actuarial Science,

University of Bern, Sidlerstrasse 5, CH-3012 Bern, Switzerland

e-mail: ilya.molchanov@stat.unibe.ch 
Most of results about random convex hulls are available in the planar case, i.e. for $d=2$. The typical questions about random polytopes $P_{n}$ concern the limit theorems for the geometric characteristics of $P_{n}$, for instance the area, the perimeter and the number of vertices of $P_{n}$, see Bräker and Hsing (1998), Groeneboom (1988), Schneider (1988). Further important results concern the quantities that characterise the worst case approximation, notably the Hausdorff distance between $K$ and $P_{n}$, see Bräker et al. (1998), Dümbgen and Walther (1996). It is well known (Schneider 1993) that the Hausdorff distance between two convex sets equals the uniform distance between their support functions defined on the unit sphere, i.e.

$$
\rho_{\mathrm{H}}\left(P_{n}, K\right)=\sup _{u:\|u\|=1}\left(h(K, u)-h\left(P_{n}, u\right)\right),
$$

where $\|u\|$ is the Euclidean norm of $u \in \mathbb{R}^{d}$,

$$
h(K, u)=\sup \{\langle u, x\rangle: x \in K\}
$$

is the support function of $K$ (and similar for $P_{n}$ ) and $\langle u, x\rangle$ is the scalar product in $\mathbb{R}^{d}$. For instance, Dümbgen and Walther (1996) shows that $\rho_{\mathrm{H}}\left(P_{n}, K\right)$ is of order $\mathcal{O}\left(\left(n^{-1} \log n\right)^{2 /(d-1)}\right)$ if the points are uniformly distributed in a sufficiently smooth $K$.

The results on the best case approximation concern the behaviour of the infimum of the difference between $h(K, u)$ and $h\left(P_{n}, u\right)$. One of the few results in this direction states that if $K$ is smooth, then $n\left(h(K, u)-h\left(P_{n}, u\right)\right.$ ) (as a stochastic process indexed by $u$ from the unit sphere $\mathbb{S}^{d-1}$ ) epi-converges in distribution to a certain process derived from the Poisson point process on $\mathbb{S}^{d-1} \times[0, \infty)$, see Molchanov (1995), and Molchanov (2005, Th. 5.3.34). The epi-convergence implies the weak convergence of infima on each compact set. In particular, $n \inf _{u \in \mathbb{S}^{d-1}}\left(h(K, u)-h\left(P_{n}, u\right)\right)$ converges in distribution to an exponentially distributed random variable, i.e. the best approximation error is of the order of $n^{-1}$. If the points are uniformly distributed in $K$, then this exponential random variable has the mean being the ratio of the volume of $K$ and its surface area, see Molchanov (2005, Ex. 5.3.35). Further results along these lines can be found in Schreiber (2003).

The best case approximation can be also studied by considering how fast the diameter of $P_{n}$, diam $P_{n}$, approximates diam $K$. By diameter we understand the maximum distance between any two points from the set. Note that diam $K$ is not necessarily equal to the diameter of the smallest ball that contains $K$, e.g. if $K$ is a triangle.

A limit theorem for the diameter of $P_{n}$ was proved in Appel et al. (2002) for uniformly distributed points in a compact set $K$ with unique longest chord (whose length is the diameter) and such that the boundary of $K$ near the endpoints of this major chord is locally defined by regularly varying functions with indices strictly larger than $\frac{1}{2}$. These assumptions are fairly restrictive and exclude a number of interesting smooth sets $K$, in particular balls and ellipsoids. 
For $K$ being the unit disk in the plane, Appel et al. (2002) provides only bounds for the limit distribution, even without proving the existence of the limit. In particular, Appel et al. (2002, Th. 4) states that

$$
\begin{aligned}
1-\exp \left\{-\frac{4 t^{5 / 2}}{3^{5 / 2} \pi}\right\} & \leq \liminf _{n \rightarrow \infty} \mathbf{P}\left\{n^{4 / 5}\left(2-\operatorname{diam} P_{n}\right) \leq t\right\} \\
& \leq \limsup _{n \rightarrow \infty} \mathbf{P}\left\{n^{4 / 5}\left(2-\operatorname{diam} P_{n}\right) \leq t\right\} \\
& \leq 1-\exp \left\{-\frac{4 t^{5 / 2}}{\pi}\right\}, \quad t>0 .
\end{aligned}
$$

In the classical theory of extreme values it is possible to consider the maximum of random samples with bounded or unbounded supports. Quite similarly, in the extreme problems for random polytopes one can consider samples supported by the whole $\mathbb{R}^{d}$ or by a compact convex subset $K$ in $\mathbb{R}^{d}$. In this paper we consider only the latter case. The limit theorems for the largest interpoint distances for samples from the whole $\mathbb{R}^{d}$ have been proved in Matthews and Rukhin (1993) for standard normally distributed samples and in Henze and Klein (1996) for more general spherically symmetric samples.

In this paper we obtain a limit law for the diameter of $P_{n}$, where $P_{n}$ is the convex hull of a sample $\Xi_{n}=\left\{\xi_{1}, \ldots, \xi_{n}\right\}$ of independent points distributed in the $d$-dimensional unit ball

$$
B=\left\{x \in \mathbb{R}^{d}:\|x\| \leq 1\right\}
$$

according to some probability measure $\kappa$. The diameter of a set $F \subset \mathbb{R}^{d}$ is its largest interpoint distance, i.e.

$$
\operatorname{diam} F=\sup _{x, y \in F}\|x-y\|,
$$

and it is obvious that the diameter of $F$ equals the diameter of its convex hull. In the special case when $\kappa$ is the uniform distribution, the following result provides a considerable improvement of Appel et al. (2002, Th. 4).

Theorem 1.1 As $n \rightarrow \infty$, the diameter of the convex hull $P_{n}$ of $n$ independent points distributed uniformly in the d-dimensional unit ball $B, d \geq 2$, has limit distribution given by

$$
\begin{gathered}
\mathbf{P}\left\{n^{\frac{4}{d+3}}\left(2-\operatorname{diam} P_{n}\right) \leq t\right\} \rightarrow 1-\exp \left\{-\frac{2^{d} d \Gamma\left(\frac{d}{2}+1\right)}{\sqrt{\pi}(d+1)(d+3) \Gamma\left(\frac{d+1}{2}\right)} t^{\frac{d+3}{2}}\right\}, \\
t>0,
\end{gathered}
$$

where $\Gamma(x)=\int_{0}^{\infty} s^{x-1} e^{-s} d s$ denotes the Gamma function.

This theorem is proved by showing that the same limit distribution is shared by the diameter of the set of points that form a homogeneous Poisson process $\Pi$ of constant intensity $\lambda=n / \mu_{d}(B)$ restricted on $B$, so that the total number 
of points in $\Pi$ has mean $n$. See Section 3 for a more general de-Poissonisation argument, which implies that the diameter of the support of a general binomial process with $n$ points and of the corresponding Poisson process share the same limiting distribution (if it exists).

The problem in dimension 1 is easy to solve, see e.g. Galambos (1978). It is interesting to note that if all $\left(\begin{array}{c}n \\ 2\end{array}\right)$ random distances $\left\|\xi_{i}-\xi_{j}\right\|$ are treated as an i.i.d. sequence of random variables with the common distribution determined by the length of the random chord in $K$, then the maximum of these distances has the same limit law as described in Theorem 1.1. This is explained by the fact that only different pairs of points contribute to diam $P_{n}$, while the probability that a point has considerably large interpoint distances with two or more other points is negligible. This argument stems from Silverman and Brown (1978) and was used in the proofs in Matthews and Rukhin (1993) and Henze and Klein (1996). Our proof relies on properties of the Poisson process with a subsequent application of the de-Poissonisation argument. Lao (2006) gave an alternative proof of Theorem 1.1 based on the results from Silverman and Brown (1978).

The paper is organised as follows. In Section 2 we establish the asymptotic behaviour of the diameter for a Poisson point process in $B$ with growing intensity. The conditions on the intensity $\kappa$ of the Poisson point process require certain asymptotic behaviour of the distance between two typical points of the process and a certain boundedness condition on $\kappa$.

In Section 4 we investigate the asymptotic behaviour of the diameter of the set of points that form the Poisson point process with intensity $n \kappa$, where $\kappa$ is a spherically symmetric distribution. Section 5 describes several examples, in particularly, where $\kappa$ is the uniform measure on $B$ and on $\mathbb{S}^{d-1}$, respectively. Further examples concern distributions which are not spherically symmetric. Note that in general it is not required that $\kappa$ is supported by a convex set.

The ball of radius $r$ centred at the origin is denoted by $r B$, so that $B$ is the unit ball. By $\mu_{d}$ we denote the $d$-dimensional Lebesgue measure in $\mathbb{R}^{d}$. Furthermore, $\mu_{d-1}$ is the surface area measure on the unit sphere $\mathbb{S}^{d-1}$. By $\kappa$ we understand a certain fixed probability measure on $B$ and $\xi_{1}, \xi_{2}, \ldots$ are i.i.d. points distributed according to $\kappa$. Finally, $\Pi_{v}$ denotes the Poisson process on $B$ of intensity measure $v$, where we write shortly $\Pi$ if no ambiguity occurs or the intensity measure is immaterial. Note that $\Pi$ is a counting measure, so that $\Pi(F)$ denotes the number of points of a point process inside a set $F$ and $\Pi(F)=0$ is equivalent to the fact that the support of $\Pi$ misses $F$. We write diam $\Pi$ for the diameter of the support of $\Pi$ and call it shortly the diameter of $\Pi$.

\section{Diameters for Poisson Processes}

Consider a Poisson process $\Pi=\Pi_{n \kappa}$ with the intensity measure proportional to a probability measure $\kappa$ on the unit ball $B$. Consider the convolution of $\kappa$ with the reflected $\kappa$, i.e. the probability measure $\tilde{\kappa}$ that determines the 
distribution of $\tilde{\xi}=\xi_{1}-\xi_{2}$ for independent $\xi_{1}$ and $\xi_{2}$ distributed according to $\kappa$. Assume throughout that the support of $\tilde{\kappa}$ contains points with norms arbitrarily close to 2, so that the diameter of $\Pi_{n \kappa}$ approaches 2 as $n \rightarrow \infty$. In this section we determine the asymptotic distribution of $2-\operatorname{diam} \Pi_{n \kappa}$ as $n \rightarrow \infty$.

For any set $F$ in $\mathbb{R}^{d}, \breve{F}$ denotes the reflected set $\{-x: x \in F\}$ and $\check{\Pi}$ is defined by $\breve{\Pi}(F)=\Pi(\breve{F})$ for all Borel $F$. Furthermore, let $\tilde{\Pi}$ be the convolution of $\Pi$ and $\check{\Pi}$, i.e. the counting measure whose atoms are located at pairwise differences of the points from $\Pi$.

The distribution of the diameter of $\Pi$ is closely related to the probability that the inner $s$-shell $2 B \backslash(2-s) B$ of the ball of radius 2 contains no points from the support of $\tilde{\Pi}$. Indeed

$$
\mathbf{P}\{\operatorname{diam} \Pi \leq 2-s\}=\mathbf{P}\{\tilde{\Pi}(2 B \backslash(2-s) B)=0\},
$$

and by the symmetry of $\tilde{\Pi}$,

$$
\mathbf{P}\{\operatorname{diam} \Pi \leq 2-s\}=\mathbf{P}\{\tilde{\Pi}((2 B \backslash(2-s) B) \cap H)=0\},
$$

where $H$ is any half-space bounded by a $(d-1)$-dimensional hyperplane passing through the origin.

For each $A \subset \mathbb{S}^{d-1}$ define

$$
A_{s}=\{r x: x \in A, r \in[2-s, 2]\} .
$$

For each point $u \in \mathbb{S}^{d-1}$ define a cap of the unit ball of height $s \in(0,1)$ by

$$
D_{s}(u)=B \cap\left\{x \in \mathbb{R}^{d}:\langle x, u\rangle \geq 1-s\right\},
$$

where $\langle x, u\rangle$ denotes the scalar product. For $A \subset \mathbb{S}^{d-1}$ define

$$
D_{s}(A)=\cup_{u \in A} D_{s}(u), \quad s \in(0,1) .
$$

The following lemma implies that $D_{s}(A)$ and $D_{s}(\check{A})$ are subsets of $B \backslash(1-s) B$ with the property that $x_{1}-x_{2} \in A_{s}$ for some $x_{1}, x_{2} \in B$ yields that $x_{1}$ belongs to $D_{s}(A)$ and $x_{2}$ to $D_{s}(\check{A})$.

Lemma 2.1 For each $A \subset \mathbb{S}^{d-1}, s \in(0,1)$ and each $x_{1} \in B \backslash D_{s}(A)$ and $x_{2} \in B$, we have $x_{1}-x_{2} \notin A_{s}$.

Proof By definition of $D_{s}(u)$ and the fact that $\left\|x_{2}\right\| \leq 1$, the inequality

$$
\left\langle u, x_{1}-x_{2}\right\rangle=\left\langle u, x_{1}\right\rangle+\left\langle u,-x_{2}\right\rangle<2-s
$$

holds for each $u \in A$. If $x_{1}-x_{2} \in A_{s}$, then $\left\|x_{1}-x_{2}\right\| \geq 2-s$ and $u_{0}=\left(x_{1}-x_{2}\right)$ $\left\|x_{1}-x_{2}\right\|^{-1} \in A$. Now write $2-s \leq\left\|x_{1}-x_{2}\right\|=\left\langle u_{0}, x_{1}-x_{2}\right\rangle$, which is a contradiction to the first inequality, and hence the claim.

Lemma 2.2 For each $s \in(0,1)$ and $A \subset \mathbb{S}^{d-1}$, the set $D_{s}(A)$ lies inside the $\sqrt{2 s}$-neighbourhood of $A$. 
Proof Consider arbitrary $u \in A$. Since

$$
\|x-u\|^{2}=\|x\|^{2}+\|u\|^{2}-2\langle x, u\rangle \leq 2-2(1-s)=2 s,
$$

every point $x \in D_{s}(u)$ is located within distance at most $\sqrt{2 s}$ from $u$.

Lemma 2.3 For any $A \subset \mathbb{S}^{d-1}$ and $s \in(0,1)$,

$$
\mathbf{P}\left\{\tilde{\Pi}\left(A_{s}\right) \geq 1\right\}=\mathbf{P}\left\{\tilde{\Pi}\left(A_{s}\right) \geq 1, \Pi\left(D_{s}(A)\right) \geq 1, \check{\Pi}\left(D_{s}(A)\right) \geq 1\right\} .
$$

If $A^{\prime}, A^{\prime \prime} \subset \mathbb{S}^{d-1}$ and

$$
D_{s}\left(\check{A}^{\prime}\right) \cap D_{s}\left(A^{\prime \prime}\right)=D_{s}\left(A^{\prime}\right) \cap D_{s}\left(\check{A}^{\prime \prime}\right)=\emptyset,
$$

then the random variables $\tilde{\Pi}\left(A_{s}^{\prime}\right)$ and $\tilde{\Pi}\left(A_{s}^{\prime \prime}\right)$ are independent.

Proof The statement follows from Lemma 2.1 and the independence property of the Poisson process.

Lemma 2.4 For each $A \subset \mathbb{S}^{d-1}$ and $0<s<1$, we have

$$
\begin{aligned}
n^{2} e^{-n(a+\check{a})} \mathbf{P}\left\{\tilde{\xi} \in A_{s}\right\} & \leq \mathbf{P}\left\{\tilde{\Pi}_{n \kappa}\left(A_{s}\right) \geq 1\right\} \\
& \leq n^{2}(1+n a \check{a}(a+\check{a})) \mathbf{P}\left\{\tilde{\xi} \in A_{s}\right\},
\end{aligned}
$$

where $a=\kappa\left(D_{s}(A)\right)$, $\check{a}=\kappa\left(D_{s}(\check{A})\right)$ and $\tilde{\xi}=\xi_{1}-\xi_{2}$ for $\xi_{1}$ and $\xi_{2}$ being independent points distributed according to $\kappa$.

Proof Note that $\zeta_{1}=\Pi\left(D_{s}(A)\right)$ and $\zeta_{2}=\Pi\left(D_{s}(\check{A})\right)$ are Poisson distributed with means na and nă respectively. By Eq. 2.3,

$$
\begin{aligned}
\mathbf{P}\left\{\tilde{\Pi}\left(A_{s}\right) \geq 1\right\} & =\mathbf{P}\left\{\tilde{\Pi}\left(A_{s}\right) \geq 1, \zeta_{1} \geq 1, \zeta_{2} \geq 1\right\} \\
& \geq \mathbf{P}\left\{\tilde{\Pi}\left(A_{s}\right)=1, \zeta_{1}=1, \zeta_{2}=1\right\} .
\end{aligned}
$$

An upper bound follows from

$$
\begin{aligned}
\mathbf{P}\left\{\tilde{\Pi}\left(A_{s}\right) \geq 1\right\} & =\mathbf{P}\left\{\tilde{\Pi}\left(A_{s}\right) \geq 1, \zeta_{1} \geq 1, \zeta_{2} \geq 1\right\} \\
& \leq \mathbf{P}\left\{\tilde{\Pi}\left(A_{s}\right)=1, \zeta_{1}=1, \zeta_{2}=1\right\}+I,
\end{aligned}
$$

where

$$
I=\sum_{\substack{k_{1}, k_{2} \geq 1 \\ \max \left(k_{1}, k_{2}\right) \geq 2}} \mathbf{P}\left\{\tilde{\Pi}\left(A_{s}\right) \geq 1, \zeta_{1}=k_{1}, \zeta_{2}=k_{2}\right\} .
$$

The subadditivity of probability and the fact that $\zeta_{1}$ and $\zeta_{2}$ are independent immediately imply that

$$
\mathbf{P}\left\{\tilde{\Pi}\left(A_{s}\right) \geq 1 \mid \zeta_{1}=k_{1}, \zeta_{2}=k_{2}\right\} \leq k_{1} k_{2} \mathbf{P}\left\{\xi_{1}-\xi_{2} \in A_{s}\right\}
$$


Thus,

$$
\begin{aligned}
I & \leq \mathbf{P}\left\{\xi_{1}-\xi_{2} \in A_{s}\right\}\left(\mathbf{E}\left(\zeta_{1} \zeta_{2}\right)-\mathbf{P}\left\{\zeta_{1}=1\right\} \mathbf{P}\left\{\zeta_{2}=1\right\}\right) \\
& =\mathbf{P}\left\{\xi_{1}-\xi_{2} \in A_{s}\right\}\left(n^{2} a \check{a}-n^{2} a \check{a} e^{-n(a+\check{a})}\right) \\
& \leq \mathbf{P}\left\{\xi_{1}-\xi_{2} \in A_{s}\right\} n^{3} a \check{a}(a+\check{a}) .
\end{aligned}
$$

Now write

$$
\begin{aligned}
\mathbf{P}\left\{\tilde{\Pi}\left(A_{s}\right)=1, \zeta_{1}=1, \zeta_{2}=1\right\} & =\mathbf{P}\left\{\tilde{\Pi}\left(A_{s}\right)=1 \mid \zeta_{1}=1, \zeta_{2}=1\right\} n^{2} a \check{a} e^{-n(a+\check{a})} \\
& =\mathbf{P}\left\{\eta_{1}-\eta_{2} \in A_{s}\right\} n^{2} a \check{a} e^{-n(a+\check{a})},
\end{aligned}
$$

where $\eta_{1}$ and $\eta_{2}$ are independent points distributed according to the normalised measure $\kappa$ restricted onto $D_{s}(A)$ and $D_{s}(\check{A})$ respectively. Because of Lemma 2.1,

$$
\mathbf{P}\left\{\eta_{1}-\eta_{2} \in A_{s}\right\}=\frac{1}{a \check{a}} \mathbf{P}\left\{\xi_{1}-\xi_{2} \in A_{s}\right\},
$$

and the proof is complete.

Let

$$
C(u, r)=\left\{x \in \mathbb{S}^{d-1}:\|x-u\| \leq r\right\}, \quad u \in \mathbb{S}^{d-1}, r>0,
$$

denote the spherical ball, i.e. the subset of $\mathbb{S}^{d-1}$ that consists of all points within distance at most $r$ from $u$. Then

$$
C_{s}(u, r)=\{a x: x \in C(u, r), a \in[2-s, 2]\}
$$

in accordance with Eq. 2.2.

Introduce the following assumption on the distribution of the difference $\tilde{\xi}$ between two independent points in $B$ distributed according to $\kappa$. Assume that for a finite non-trivial measure $\sigma$ on $\mathbb{S}^{d-1}$, some $\gamma>0$ and $\left[\delta^{\prime}, \delta^{\prime \prime}\right] \subset\left(0, \frac{1}{2}\right)$ we have

$$
\lim _{s \downarrow 0} \frac{\mathbf{P}\left\{\tilde{\xi} \in C_{s}\left(u, z_{s}\right)\right\}}{s^{\gamma} \sigma\left(C\left(u, z_{s}\right)\right)}=1
$$

and

$$
\lim _{s \downarrow 0} s^{-\gamma / 2} \kappa\left(D_{s}\left(C\left(u, z_{s}\right)\right)\right)=0
$$

uniformly in $u \in \mathbb{S}^{d-1}$ and $z_{s} \in\left[s^{\delta^{\prime \prime}}, s^{\delta^{\prime}}\right]$. If $u$ does not belong to the support of $\sigma$, then the denominator in Eq. 2.4 equals zero for all sufficiently small $s$, and Eq. 2.4 is understood as the fact that the numerator also equals zero for all sufficiently small $s$. Since $\tilde{\xi}$ has a centrally symmetric distribution, the measure $\sigma$ is necessarily centrally symmetric.

Lemma 2.5 If Eq. 2.4 holds with $\gamma<d+1, \kappa$ is absolutely continuous on $B \backslash(1-s) B$ for some $s>0$ and possesses there a bounded density, then Eq. 2.5 holds with

$$
\frac{\gamma-2}{2(d-1)}<\delta^{\prime} \leq \delta^{\prime \prime}<\frac{1}{2} .
$$


Proof It suffices to show that, for any given $u \in \mathbb{S}^{d-1}$,

$$
\lim _{s \downarrow 0} s^{-\gamma / 2} \mu_{d}\left(D_{s}\left(C\left(u, s^{\delta}\right)\right)\right)=0 .
$$

By Lemma 2.2, noticing that $\delta<\frac{1}{2}$, this would follow from

$$
s^{-\gamma / 2} \mu_{d-1}\left(C\left(u, 2 s^{\delta}\right)\right) s \rightarrow 0 \quad \text { as } s \downarrow 0 .
$$

The latter is justified by Eq. 2.4, since $-\frac{1}{2} \gamma+\delta(d-1)+1>0$ for all $\delta \in$ $\left[\delta^{\prime}, \delta^{\prime \prime}\right]$. Finally, $\gamma<d+1$ implies that $\frac{\gamma-2}{2(d-1)}<\frac{1}{2}$, so that Eq. 2.6 makes sense.

In general, Eq. 2.5 is weaker than the boundedness of the density of $\kappa$ with respect to the Lebesgue measure, which, e.g., excludes the case of $\kappa$ supported by $\mathbb{S}^{d-1}$.

Lemma 2.6 Let Eqs. 2.4 and 2.5 hold, and let $\delta \in\left[\delta^{\prime}, \delta^{\prime \prime}\right]$. Then, for each $t>0$ and $n=t s^{-\gamma / 2}$,

$$
\mathbf{P}\left\{\tilde{\Pi}_{n \kappa}\left(A_{s}\right) \geq 1\right\} \leq c t^{2} \sigma\left(A^{s^{\delta}}\right),
$$

holds with a certain constant $c$ for all sufficiently small $s>0$ uniformly over all measurable $A \subset \mathbb{S}^{d-1}$.

Proof By Besicovitch's covering theorem (Mattila 1995, Th. 2.7), it is possible to cover the unit sphere with spherical balls $C\left(u_{i}, s^{\delta}\right), i=1, \ldots, m$, of diameter $s^{\delta}$, such that each point is covered at most $p_{d}$ times for a certain constant $p_{d}$.

By the choice of $n$, Lemma 2.4 and Eq. 2.4,

$$
\begin{aligned}
\mathbf{P}\left\{\tilde{\Pi}_{n \kappa}\left(C_{s}\left(u_{i}, s^{\delta}\right)\right) \geq 1\right\} & \leq t^{2} s^{-\gamma} \mathbf{P}\left\{\tilde{\xi} \in C_{s}\left(u_{i}, s^{\delta}\right)\right\}\left(1+n a_{i} \check{a}_{i}\right) \\
& \leq t^{2}(1+\varepsilon) \sigma\left(C\left(u_{i}, s^{\delta}\right)\right)\left(1+n a_{i} \check{a}_{i}\right)
\end{aligned}
$$

for any $\varepsilon>0$ and all sufficiently small $s$, where $a_{i}=\kappa\left(D_{s}\left(C\left(u_{i}, s^{\delta}\right)\right)\right)$ and $\check{a}_{i}=\kappa\left(D_{s}\left(C\left(-u_{i}, s^{\delta}\right)\right)\right)$. Condition 2.5 implies that $n a_{i} \check{a}_{i} \rightarrow 0$ as $s \downarrow 0$ uniformly in $u_{i}$. Therefore,

$$
\mathbf{P}\left\{\tilde{\Pi}_{n \kappa}\left(C_{s}\left(u_{i}, s^{\delta}\right)\right) \geq 1\right\} \leq t^{2}(1+\varepsilon)^{2} \sigma\left(C\left(u_{i}, s^{\delta}\right)\right)
$$

for all sufficiently small $s$.

If $I_{A}$ is the subset of $\{1, \ldots, m\}$ such that $C\left(u_{i}, s^{\delta}\right) \cap A \neq \emptyset$ for $i \in I_{A}$, then

$$
\begin{aligned}
\mathbf{P}\left\{\tilde{\Pi}_{n \kappa}\left(A_{s}\right) \geq 1\right\} & \leq \sum_{i \in I_{A}} \mathbf{P}\left\{\tilde{\Pi}_{n \kappa}\left(C_{s}\left(u_{i}, s^{\delta}\right)\right) \geq 1\right\} \\
& \leq t^{2}(1+\varepsilon)^{2} \sum_{i \in I_{A}} \sigma\left(C\left(u_{i}, s^{\delta}\right)\right) \\
& \leq p_{d} t^{2}(1+\varepsilon)^{2} \sigma\left(A^{s^{\delta}}\right)
\end{aligned}
$$

for all sufficiently small $s$, so that Eq. 2.7 holds with $c=p_{d}(1+\varepsilon)^{2}$. 
We often impose the following assumption on $\sigma$ :

(S) $\sigma$ is a finite measure on $\mathbb{S}^{d-1}$ such that

$$
\sigma(A) \leq f\left(\mu_{d-1}(A)\right)
$$

for all measurable $A \subset \mathbb{S}^{d-1}$ with a function $f$ such that $f(x) \rightarrow 0$ as $x \downarrow 0$.

It is easy to see that Eq. 2.8 holds if $\sigma$ is absolutely continuous with respect to $\mu_{d-1}$ and has a bounded density. An atomic $\sigma$ clearly violates (S).

Theorem 2.7 Assume that Eqs. 2.4 and 2.5 hold with $\delta^{\prime}<\delta^{\prime \prime}$ and a $\sigma$ that satisfies $(\boldsymbol{S})$. Then

$$
\lim _{n \rightarrow \infty} \mathbf{P}\left\{n^{2 / \gamma}\left(2-\operatorname{diam} \Pi_{n \kappa}\right) \leq t\right\}=1-e^{-\frac{1}{2} t^{\gamma} \sigma_{0}}, \quad t \geq 0,
$$

where $\sigma_{0}=\sigma\left(\mathbb{S}^{d-1}\right)$.

Proof Let $\mathbb{S}_{+}^{d-1}$ denote the half-sphere, obtained by intersecting $\mathbb{S}^{d-1}$ with any fixed half-space $H$. Fix any $\varepsilon>0$ and consider disjoint spherical balls $C\left(x_{i}, s^{\delta_{i}}\right)$, $i=1, \ldots, m$, where $x_{i} \in \mathbb{S}_{+}^{d-1}$ and $\delta_{i} \in\left[\delta^{\prime}, \delta^{*}\right]$ for any fixed $\delta^{*} \in\left(\delta^{\prime}, \delta^{\prime \prime}\right)$. Since these spherical balls are constructed using varying scales of $s$, it is possible to pack them arbitrarily dense as $s \downarrow 0$, i.e. assume that the Lebesgue measure of the uncovered part is smaller than $\varepsilon$. This can be seen by constructing such families of balls that cover at least the $(1-\varepsilon)$ th part of the Euclidean space and then using the fact that an arbitrarily large part of the unit sphere can be realised as a bi-Lipschitz map from the Euclidean space.

Define the spherical balls

$$
A^{(i)}=C\left(x_{i}, s^{\delta_{i}}-\sqrt{2 s}\right), \quad i=1, \ldots, m .
$$

Since $\sqrt{2 s} \leq s^{\delta_{i}}$ for all sufficiently small $s$, Lemma 2.2 implies that $D_{s}\left(A^{(i)}\right)$, $i=1, \ldots, m$, are pairwise disjoint and nonempty for all sufficiently small $s$. By Lemma 2.3 , the random variables $\tilde{\Pi}\left(A_{s}^{(i)}\right), i=1, \ldots, m$, are independent.

Denote

$$
\Delta(s)=\mathbb{S}_{+}^{d-1} \backslash\left(A^{(1)} \cup \cdots \cup A^{(m)}\right)
$$

to be the uncovered part of $\mathbb{S}_{+}^{d-1}$ left by the $A^{(i)}$ 's. Let $\Delta\left(s ; s^{\delta^{\prime \prime}}\right)$ denote the $s^{\delta^{\prime \prime}}$-neighbourhood of $\Delta(s)$ on the sphere. The $(d-1)$-dimensional Lebesgue measure of $\Delta\left(s ; s^{\delta^{\prime \prime}}\right)$ is bounded from above by the sum of the $\mu_{d-1}$-measure of 
the part left uncovered by $C\left(x_{i}, s^{\delta_{i}}\right), i=1, \ldots, m$, and the sum of the measures of $C\left(x_{i}, s^{\delta_{i}}\right) \backslash C\left(x_{i}, s^{\delta_{i}}-\sqrt{2 s}-s^{\delta^{\prime \prime}}\right)$ for $i=1, \ldots, m$. Thus

$$
\begin{aligned}
\mu_{d-1}\left(\Delta\left(s ; s^{\delta^{\prime \prime}}\right)\right) & \leq \varepsilon+\sum_{i=1}^{m} c_{1} s^{\delta_{i}(d-2)}\left(\sqrt{2 s}+s^{\delta^{\prime \prime}}\right) \\
& \leq \varepsilon+\sum_{i=1}^{m} c_{1} s^{\delta_{i}(d-1)} s^{-\delta^{*}}\left(\sqrt{2 s}+s^{\delta^{\prime \prime}}\right) \\
& \leq \varepsilon+c_{2} s^{-\delta^{*}}\left(\sqrt{2 s}+s^{\delta^{\prime \prime}}\right) \leq 2 \varepsilon
\end{aligned}
$$

for all sufficiently small $s$, where $c_{1}$ and $c_{2}$ are positive constants. Condition (S) implies that $\sigma\left(\Delta\left(s ; s^{\delta^{\prime \prime}}\right)\right)$ is smaller than $f(2 \varepsilon)$ for all sufficiently small $s$. In turn, $f(2 \varepsilon)$ can be made smaller than any given $\varepsilon^{\prime}>0$.

For any fixed $t>0$ consider the Poisson process $\Pi$ with intensity measure $n \kappa$ with $n=t s^{-\gamma / 2}$ for a fixed $t$. By Lemma 2.6,

$$
\lim _{s \downarrow 0} \mathbf{P}\left\{\tilde{\Pi}\left(\Delta_{s}(s)\right) \geq 1\right\} \leq c t^{2} \varepsilon^{\prime} .
$$

By Eq. 2.1,

$$
\mathbf{P}\{\operatorname{diam} \Pi \leq 2-s\}=\mathbf{P}\left\{\tilde{\Pi}\left(A_{s}^{(i)}\right)=0, i=1, \ldots, m, \tilde{\Pi}\left(\Delta_{s}(s)\right)=0\right\} .
$$

By the independence of $\tilde{\Pi}\left(A_{s}^{(i)}\right), i=1, \ldots, m$,

$$
I \leq \mathbf{P}\{2-\operatorname{diam} \Pi \leq s\} \leq I+\mathbf{P}\left\{\tilde{\Pi}\left(\Delta_{s}(s)\right) \geq 1\right\},
$$

where

$$
I=1-\prod_{i=1}^{m} \mathbf{P}\left\{\tilde{\Pi}\left(A_{s}^{(i)}\right)=0\right\} .
$$

By Lemma 2.4,

$$
\begin{aligned}
\prod_{i=1}^{m}\left(1-n^{2}\left(1+y_{1}(s)\right) \mathbf{P}\left\{\tilde{\xi} \in A_{s}^{(i)}\right\}\right) & \leq \prod_{i=1}^{m} \mathbf{P}\left\{\tilde{\Pi}\left(A_{s}^{(i)}\right)=0\right\} \\
& \leq \prod_{i=1}^{m}\left(1-n^{2} e^{-2 y_{2}(s)} \mathbf{P}\left\{\tilde{\xi} \in A_{s}^{(i)}\right\}\right)
\end{aligned}
$$

where

$$
\begin{aligned}
& y_{1}(s)=\max _{1 \leq i \leq m} n \kappa\left(D_{s}\left(A^{(i)}\right)\right) \kappa\left(D_{s}\left(\check{A}^{(i)}\right)\right), \\
& y_{2}(s)=\max _{1 \leq i \leq m} n \kappa\left(D_{s}\left(A^{(i)}\right)\right) .
\end{aligned}
$$


By Eq. 2.5, $y_{2}(s)$ (and thereupon also $y_{1}(s)$ ) converge to zero as $s \downarrow 0$ for $n=t s^{-\gamma / 2}$. By Eq. 2.4 with $z_{s}=s^{\delta_{i}}-\sqrt{2 s}$,

$$
\frac{n^{2} \mathbf{P}\left\{\tilde{\xi} \in A_{s}^{(i)}\right\}}{\sigma\left(A^{(i)}\right)} \rightarrow t^{\gamma} \quad \text { as } s \downarrow 0 .
$$

Since $y_{1}(s) \rightarrow 0$ and $y_{2}(s) \rightarrow 0$,

$$
\lim _{s \downarrow 0} \prod_{i=1}^{m}\left(1-n^{2}\left(1+y_{1}(s)\right) \mathbf{P}\left\{\tilde{\xi} \in A_{s}^{(i)}\right\}\right)=\lim _{s \downarrow 0} \prod_{i=1}^{m}\left(1-t^{\gamma} \sigma\left(A^{(i)}\right)\right),
$$

and

$$
\lim _{s \downarrow 0} \prod_{i=1}^{m}\left(1-n^{2} e^{-2 y_{2}(s)} \mathbf{P}\left\{\tilde{\xi} \in A_{s}^{(i)}\right\}\right)=\lim _{s \downarrow 0} \prod_{i=1}^{m}\left(1-t^{\gamma} \sigma\left(A^{(i)}\right)\right) .
$$

By taking logarithms, and using the inequality $|\log (1+x)-x| \leq x^{2}$ for $|x|<1$, we see that

$$
\begin{aligned}
\lim _{s \downarrow 0} \prod_{i=1}^{m}\left(1-t^{\gamma} \sigma\left(A^{(i)}\right)\right) & =\exp \left\{-t^{\gamma} \lim _{s \downarrow 0} \sum_{i=1}^{m} \sigma\left(A^{(i)}\right)\right\} \\
& =\exp \left\{-\frac{1}{2} t^{\gamma} \sigma_{0}\right\} .
\end{aligned}
$$

For this, note that $\sigma$ is necessarily symmetric, so that $\sigma\left(\mathbb{S}_{+}^{d-1}\right)=\sigma_{0} / 2$. Finally, Eq. 2.9 is obtained from Eq. 2.11 and the choice of $n=t s^{-\gamma / 2}$.

Instead of imposing (S) it is possible to require that for every $s>0$ there exists a covering of $\mathbb{S}^{d-1}$ by spherical balls $C\left(x_{i}, s^{\delta_{i}}\right)$ of radii $s^{\delta_{i}}$ with $\delta_{i} \in$ $\left[\delta^{\prime}, \delta^{\prime \prime}\right] \subset\left(0, \frac{1}{2}\right)$ such that $\sigma\left(\Delta\left(s ; s^{\delta^{\prime \prime}}\right)\right) \rightarrow 0$ as $s \downarrow 0$, where $\Delta(s)$ is given by (2.10). Since this condition always holds in dimension $d=2$ with $\delta^{\prime}=\delta^{\prime \prime}$, we obtain the following result for interpoint distances in the unit disk.

Theorem 2.8 Assume that $d=2$ and Eqs. 2.4 and 2.5 hold with $z_{s}=s^{\delta}$ for some fixed $\delta \in\left(0, \frac{1}{2}\right)$ uniformly over $u \in \mathbb{S}^{1}$. Then Eq. 2.9 holds.

Furthermore, (S) can be relaxed for $\sigma$ supported by a lower-dimensional part of the unit sphere, for instance, obtained as the intersection of $\mathbb{S}^{d-1}$ with a certain hyperplane. Then in Eq. 2.8 one has to consider the Hausdorff measure of $A$ of a lower order than $(d-1)$.

Finally, note that if $\sigma_{1}, \sigma_{2}, \ldots$ satisfy (S) and have total mass bounded by a fixed number then $\sup p_{i} \sigma_{i}$ also satisfies (S) if $\sum p_{i}<\infty$. This construction is useful if $\sigma$ is supported by a countable family of great circles on the unit sphere.

Instead of imposing Eqs. 2.4 and 2.5, it is possible to deduce the limiting distribution in Eq. 2.9 using a direct assumption on the asymptotic distribution of $\tilde{\Pi}_{n \kappa}$ and assuming (S) only for the non-atomic part of $\sigma$. 
Theorem 2.9 Assume that, for $\left[\delta^{\prime}, \delta^{\prime \prime}\right] \subset\left(0, \frac{1}{2}\right)$ with $\delta^{\prime}<\delta^{\prime \prime}$,

$$
\lim _{s \downarrow 0} \frac{\mathbf{P}\left\{\tilde{\Pi}_{n \kappa}\left(C_{s}\left(u, z_{s}\right)\right) \geq 1\right\}}{\sigma\left(C\left(u, z_{s}\right)\right)}=g(t, u)
$$

for some function $g$ uniformly in $u \in \mathbb{S}^{d-1}$ and $z_{s} \in\left[\delta^{\delta^{\prime \prime}}, s^{\delta^{\prime}}\right]$, where $n=(t / s)^{\gamma / 2}$ and $\sigma$ is a finite measure on $\mathbb{S}^{d-1}$. If the non-atomic part $\sigma^{\prime}$ of $\sigma$ satisfies $(\boldsymbol{S})$, then

$$
\begin{aligned}
& \lim _{n \rightarrow \infty} \mathbf{P}\left\{n^{2 / \gamma}\left(2-\operatorname{diam} \Pi_{n \kappa}\right) \leq t\right\} \\
& \quad=1-\exp \left\{-\frac{1}{2} \int_{\mathbb{S}^{d-1}} g(t, u) \sigma^{\prime}(d u)\right\} \prod_{\substack{x_{i} \in \mathbb{S}^{d-1} \\
\sigma\left(\left\{x_{i}\right\}\right)>0}}\left(1-g\left(t, x_{i}\right) \sigma\left(\left\{x_{i}\right\}\right)\right)^{\frac{1}{2}}
\end{aligned}
$$

for all $t \geq 0$.

Proof For the proof we use the same partition of the unit sphere and the sets $A^{(i)}$ as in the proof of Theorem 2.7. If $\sigma$ has an atomic part, choose the points $x_{1}, \ldots, x_{m}$ in such a way that they have so many atoms of $\sigma$ among them that the total $\sigma$-content of the remaining atoms is less than $\varepsilon$.

In the remainder of the proof we need to split the product in the left-hand side of Eq. 2.12 into the factors that correspond to the non-atomic and the atomic parts of $\sigma$. Notice that Lemma 2.4 is no longer needed to derive the asymptotics for $\mathbf{P}\left\{\tilde{\Pi}\left(A_{s}^{(i)}\right)=0\right\}$ from the distribution of $\tilde{\xi}$. The square root of the product in Eq. 2.14 appears because the atoms of $\sigma$ form centrally symmetric pairs of points.

The cases when $\sigma$ has atoms often appear if $\kappa$ is supported by a subset $K$ of $B$ and such that $K$ is sufficiently "sharply pointed" near the points where its diameter is achieved. The typical example of such $K$ is a segment, see Section 5.5. Other such examples appear if $K$ satisfies the conditions imposed in Appel et al. (2002).

Note that the limiting distribution of the diameter remains unchanged if $\kappa$ is arbitrarily changed inside $s B$ for any fixed $s<1$.

\section{De-Poissonisation}

Let $\Pi$ be the Poisson process with intensity measure $n \kappa$. Given $\Pi(K)=n$, the distribution of $\Pi$ coincides with the distribution of $\Xi_{n}=\left\{\xi_{1}, \ldots, \xi_{n}\right\}$ being the binomial process on $K$, i.e. $n$ i.i.d. points sampled from $\kappa$. In the other direction, the distribution of $\Pi$ coincides with the distribution of $\Xi_{N}$, where $N$ is the Poisson random variable of mean $n$ independent of the i.i.d. points $\xi_{i}$ 's distributed according to $\kappa$. This simple relationship makes it possible to use the de-Poissonisation technique (Penrose 2003) in order to obtain the limit theorem for functionals of $\Xi_{n}$. The key issue that simplifies our proofs is the monotonicity of the diameter functional. Indeed, the diameter of $\Xi_{n}$ is stochastically greater than the diameter of $\Xi_{m}$ for $n>m$. Another useful tool is provided by the following lemma from Penrose (2003, p. 18). 
Lemma 3.1 Let $N$ be a Poisson random variable with mean $\lambda$. For every $\gamma>0$ there exists a constant $\lambda_{1}=\lambda_{1}(\gamma) \geq 0$ such that

$$
\mathbf{P}\left\{|N-\lambda| \geq \frac{1}{2} \lambda^{\frac{1}{2}+\gamma}\right\} \leq 2 \exp \left\{-\frac{1}{9} \lambda^{2 \gamma}\right\}
$$

for all $\lambda>\lambda_{1}$.

Theorem 3.2 Let $\Psi: \mathcal{N} \rightarrow \mathbb{R}$ be a non-increasing (resp. non-decreasing) functional defined on the space $\mathcal{N}$ of counting measures on $\mathbb{R}^{d}$. Furthermore, let $\Pi_{n \kappa}$ be a Poisson process with intensity measure $n \kappa$ where $\kappa$ is a probability measure on $\mathbb{R}^{d}$. If, for some non-decreasing (resp. non-increasing) sequence $a_{n}$ satisfying

$$
\lim _{n \rightarrow \infty} \frac{a_{\left[n\left(1 \pm n^{-\beta}\right)\right]}}{a_{n}}=1
$$

for some $\beta \in\left(0, \frac{1}{2}\right)$, the random variable $a_{n} \Psi\left(\Pi_{n \kappa}\right)$ converges in distribution to a random variable with cumulative distribution function $F$, then the distribution of $a_{n} \Psi\left(\Xi_{n}\right)$ also weakly converges to $F$, where $\Xi_{n}$ is a binomial process of $n$ i.i.d. points with common distribution $\kappa$.

Proof Without loss of generality assume that $\Psi$ is non-increasing. Define $\gamma=\frac{1}{2}-\beta$ and $\varepsilon_{n}=n^{-\beta}$ for some $\beta \in\left(0, \frac{1}{2}\right)$. By Lemma 3.1 and the monotonicity of $\Psi$,

$$
\begin{aligned}
\mathbf{P}\left\{\Psi\left(\Pi_{n \kappa}\right) \leq s\right\} & \leq \mathbf{P}\left\{\Psi\left(\Pi_{n \kappa}\right) \leq s,|N-n| \leq n \varepsilon_{n}\right\}+\mathbf{P}\left\{|N-n|>n \varepsilon_{n}\right\} \\
& \leq \mathbf{P}\left\{\Psi\left(\Xi_{\left[n\left(1+\varepsilon_{n}\right)\right]}\right) \leq s\right\}+2 \exp \left\{-\frac{1}{9}(2 n)^{2 \gamma}\right\} .
\end{aligned}
$$

for sufficiently large $n$. Now Eq. 3.1 yields that

$$
\begin{aligned}
\lim _{n \rightarrow \infty} \mathbf{P}\left\{a_{n} \Psi\left(\Xi_{n}\right) \leq t\right\} & =\lim _{n \rightarrow \infty} \mathbf{P}\left\{a_{\left[n\left(1+\varepsilon_{n}\right)\right]} \Psi\left(\Xi_{\left[n\left(1+\varepsilon_{n}\right)\right]}\right) \leq t\right\} \\
& \geq \lim _{n \rightarrow \infty} \mathbf{P}\left\{a_{n} \Psi\left(\Xi_{\left[n\left(1+\varepsilon_{n}\right)\right]}\right) \leq t-\varepsilon\right\}
\end{aligned}
$$

for any $\varepsilon>0$. By Eq. 3.2,

$$
\begin{aligned}
\lim _{n \rightarrow \infty} \mathbf{P}\left\{a_{n} \Psi\left(\Xi_{n}\right) \leq t\right\} & \geq \lim _{n \rightarrow \infty} \mathbf{P}\left\{a_{n} \Psi\left(\Pi_{n \kappa}\right) \leq t-\varepsilon\right\}-2 \exp \left\{-\frac{1}{9}(2 n)^{2 \gamma}\right\} \\
& =F(t-\varepsilon) .
\end{aligned}
$$

By similar calculations and

$$
\begin{aligned}
\mathbf{P}\{\Psi(\Pi) \leq s\} & \geq \mathbf{P}\left\{\Psi\left(\Xi_{\left[n\left(1-\varepsilon_{n}\right)\right]}\right) \leq s\right\} \mathbf{P}\left\{|N-n| \leq n \varepsilon_{n}\right\} \\
& \geq \mathbf{P}\left\{\Psi\left(\Xi_{\left[n\left(1-\varepsilon_{n}\right)\right]}\right) \leq s\right\}-2 \exp \left\{-\frac{1}{9}(2 n)^{-2 \gamma}\right\},
\end{aligned}
$$


we get

$$
\lim _{n \rightarrow \infty} \mathbf{P}\left\{a_{n} \Psi\left(\Xi_{n}\right) \leq t\right\} \leq F(t+\varepsilon) .
$$

The proof is finished by noticing that $\varepsilon>0$ is arbitrary and $t$ can be chosen to be any continuity point of $F$.

In particular, Theorem 3.2 is applicable for the functional $\Psi\left(\Xi_{n}\right)=$ 2 - diam $\Xi_{n}$, so that all results available for diameters of Poisson processes can be immediately reformulated for binomial processes. Note that Assumption 3.1 is e.g. fulfilled for $a_{n}=c n^{\alpha}$ with any $c>0$ and $\alpha \in \mathbb{R}$.

\section{Spherically Symmetric Distributions}

Let $\xi_{1}, \ldots, \xi_{n}$ be independent points distributed according to a spherically symmetric (also called "isotropic") distribution $\kappa$ restricted on B. Spherically symmetric distributions are closed with respect to convolution, so that $\tilde{\xi}=\xi_{1}-\xi_{2}$ is spherically symmetric too. Since $\|\tilde{\xi}\|$ and $\tilde{\xi} /\|\tilde{\xi}\|$ are independent (see e.g. Gneiting 1998),

$$
\mathbf{P}\left\{\tilde{\xi} \in A_{s}\right\}=\mathbf{P}\{\|\tilde{\xi}\| \geq 2-s\} \frac{\mu_{d-1}(A)}{\mu_{d-1}\left(\mathbb{S}^{d-1}\right)} .
$$

holds for any measurable $A \subset \mathbb{S}^{d-1}$. Therefore Eq. 2.4 is fulfilled if, for some $\gamma>0$

$$
\lim _{s \rightarrow 0} \mathbf{P}\{\|\tilde{\xi}\| \geq 2-s\} s^{-\gamma}=\sigma_{0} \in(0, \infty),
$$

where $\sigma_{0}$ then becomes the total mass of $\sigma$, so that $\sigma$ is the surface area measure on $\mathbb{S}^{d-1}$ normalised to have the total mass $\sigma_{0}$.

Furthermore, Eq. 2.5 holds if

$$
\lim _{s \downarrow 0} s^{\delta(d-1)-\gamma / 2} \mathbf{P}\{\eta \leq s\}=0,
$$

where $\eta=1-\left\|\xi_{1}\right\|$.

Lemma 4.1 If $\eta_{1}$ and $\eta_{2}$ are independent random variables distributed as $1-\left\|\xi_{1}\right\|$ and $\zeta=\eta_{1}+\eta_{2}$, then

$$
\lim _{s \downarrow 0} \frac{\mathbf{P}\{\|\tilde{\xi}\| \geq 2-s\}}{\mathbf{E}\left((s-\zeta)^{(d-1) / 2} \mathbf{1}_{\zeta \leq s}\right)}=\frac{2^{d-1} \Gamma\left(\frac{d}{2}\right)}{(d-1) \pi^{\frac{1}{2}} \Gamma\left(\frac{d-1}{2}\right)} .
$$

Proof By the cosine theorem and the fact that $\tilde{\xi}$ has the same distribution as $\xi_{1}+\xi_{2}$, we write

$$
\mathbf{P}\{\|\tilde{\xi}\| \geq 2-s\}=\mathbf{P}\left\{\left\|\xi_{1}\right\|^{2}+\left\|\xi_{2}\right\|^{2}+2\left\|\xi_{1}\right\|\left\|\xi_{2}\right\| \cos \beta \geq(2-s)^{2}\right\},
$$

where $\beta$ denotes the angle between $\xi_{1}$ and $\xi_{2}$. Hence,

$$
\mathbf{P}\{\|\tilde{\xi}\| \geq 2-s\}=\mathbf{P}\{\cos \beta \geq 1-q\},
$$


where

$$
q=\frac{(2-\zeta)^{2}-(2-s)^{2}}{2\left\|\xi_{1}\right\|\left\|\xi_{2}\right\|}
$$

If $q \geq 0$ (i.e. $\zeta \leq s)$

$$
\mathbf{P}\{\|\tilde{\xi}\| \geq 2-s\}=\frac{1}{2} \mathbf{P}\left\{\cos ^{2} \beta \geq(1-q)^{2}, \zeta \leq s\right\}=\frac{1}{2} \mathbf{E}\left(\int_{(1-q)^{2}}^{1} f(t) d t \mathbf{1}_{\zeta \leq s}\right),
$$

where the probability density function

$$
f(t)=\frac{\Gamma\left(\frac{d}{2}\right)}{\pi^{\frac{1}{2}} \Gamma\left(\frac{d-1}{2}\right)} t^{-\frac{1}{2}}(1-t)^{\frac{d-3}{2}}, \quad t \in[0,1],
$$

of $\cos ^{2} \beta$ corresponds to the Beta-distribution with parameters $\frac{1}{2}$ and $(d-1) / 2$, see Matthews and Rukhin (1993, Prop. 2). Substituting $x=1-t$ leads to

$$
\mathbf{P}\{\|\tilde{\xi}\| \geq 2-s\}=c_{1} \mathbf{E}\left(\int_{0}^{2 q\left(1-\frac{q}{2}\right)}(1-x)^{-\frac{1}{2}} x^{\frac{d-1}{2}-1} d x \mathbf{1}_{\zeta \leq s}\right),
$$

where

$$
c_{1}=\frac{1}{2} \frac{\Gamma\left(\frac{d}{2}\right)}{\pi^{\frac{1}{2}} \Gamma\left(\frac{d-1}{2}\right)} .
$$

The inequality

$$
1 \leq(1-x)^{-\frac{1}{2}} \leq(1-q)^{-1} \leq\left((1-s)^{2}-2 s\right)^{-1}
$$

leads to the bounds

$$
c_{1} \mathbf{E}\left(I \mathbf{1}_{\zeta \leq s}\right) \leq \mathbf{P}\{\|\tilde{\xi}\| \geq 2-s\} \leq c_{1}\left((1-s)^{2}-2 s\right)^{-1} \mathbf{E}\left(I \mathbf{1}_{\zeta \leq s}\right),
$$

where

$$
I=\int_{0}^{2 q\left(1-\frac{q}{2}\right)} x^{\frac{d-1}{2}-1} d x=\frac{2}{d-1}(2 q)^{\frac{d-1}{2}}\left(1-\frac{q}{2}\right)^{\frac{d-1}{2}} .
$$

By the fact that

$$
1 \leq\left(\left\|\xi_{1}\right\|\left\|\xi_{2}\right\|\right)^{-1} \leq(1-s)^{-2}
$$

and

$$
1-\frac{s}{(1-s)^{2}} \leq 1-\frac{q}{2} \leq 1,
$$

we further get the bounds

$$
\begin{gathered}
\frac{2}{d-1}\left((2-\zeta)^{2}-(2-s)^{2}\right)^{\frac{d-1}{2}}\left(1-\frac{s}{(1-s)^{2}}\right)^{\frac{d-1}{2}} \leq I \\
\leq \frac{2}{d-1}\left((2-\zeta)^{2}-(2-s)^{2}\right)^{\frac{d-1}{2}}(1-s)^{-(d-1)} .
\end{gathered}
$$


Since

$$
(4-2 s)(s-\zeta) \leq(2-\zeta)^{2}-(2-s)^{2} \leq 4(s-\zeta),
$$

the following bounds for $I$ hold

$$
\begin{aligned}
& \frac{2^{d}}{d-1}(s-\zeta)^{\frac{d-1}{2}}\left(1-\frac{s}{(1-s)^{2}}\right)^{\frac{d-1}{2}}\left(1-\frac{s}{2}\right)^{\frac{d-1}{2}} \leq I \\
& \quad \leq \frac{2^{d}}{d-1}(s-\zeta)^{\frac{d-1}{2}}(1-s)^{-(d-1)} .
\end{aligned}
$$

Plugging these bounds in Eq. 4.4 yields the result.

The following result settles the case when the density of $\eta$ is equivalent to a power function for small arguments.

Theorem 4.2 Assume that $d \geq 2$ and for some $\alpha \geq 0$ the cumulative distribution function $F(x)=\mathbf{P}\{\eta \leq x\}$ of $\eta=1-\|\xi\|$ satisfies

$$
\lim _{s \downarrow 0} s^{-\alpha} F(s)=a \in(0, \infty) .
$$

Then

$$
\lim _{n \rightarrow \infty} \mathbf{P}\left\{n^{2 / \gamma}\left(2-\operatorname{diam} \Pi_{n \kappa}\right) \leq t\right\}=1-e^{-\frac{1}{2} t^{\gamma} \sigma_{0}}, \quad t \geq 0,
$$

where $\gamma=\frac{1}{2}(d-1)+2 \alpha$ and

$$
\sigma_{0}=a^{2} c \frac{\Gamma(\alpha+1)^{2} \Gamma\left(\frac{1}{2}(d+1)\right)}{\Gamma\left(2 \alpha+\frac{1}{2}(d+1)\right)}
$$

with $c$ given by the right-hand side of Eq. 4.3.

Proof The integration by parts leads to

$$
\begin{aligned}
& \mathbf{E}\left((s-\zeta)^{(d-1) / 2} \mathbf{1}_{\zeta \leq s}\right) \\
& \quad=F(0)^{2} s^{(d-1) / 2}+\frac{(d-1)(d-3)}{4} \int_{0}^{s} \int_{0}^{s-x_{1}} F\left(x_{1}\right) F\left(x_{2}\right)\left(s-x_{1}-x_{2}\right)^{(d-5) / 2} d x_{1} d x_{2} .
\end{aligned}
$$

If $F(0)>0$, then (4.5) implies that $\alpha=0$, so that Eq. 4.1 holds with $\gamma=\frac{1}{2}(d-1)$ and $\sigma_{0}=F(0)^{2} c=a^{2} c$ by Lemma 4.1, which corresponds to Eq. 4.7.

If $F(0)=0$, then Eq. 4.5 yields that $\mathbf{E}\left((s-\zeta)^{(d-1) / 2} \mathbf{1}_{\zeta \leq s}\right)$ is equivalent as $s \downarrow 0$ to

$$
\begin{aligned}
s^{\gamma} & a^{2} \frac{(d-1)(d-3)}{4} \int_{0}^{1} \int_{0}^{1-t_{1}} t_{1}^{\alpha} t_{2}^{\alpha}\left(1-t_{1}-t_{2}\right)^{(d-5) / 2} d t_{1} d t_{2} \\
& =s^{\gamma} a^{2} \frac{(d-1)(d-3)}{4} \mathrm{~B}\left(\alpha+1, \alpha+\frac{d-1}{2}\right) \mathrm{B}\left(\alpha+1, \frac{d-3}{2}\right) \\
& =s^{\gamma} a^{2} \alpha^{2} \Gamma(\alpha)^{2} \frac{\Gamma\left(\frac{1}{2}(d+1)\right)}{\Gamma\left(2 \alpha+\frac{1}{2}(d+1)\right)}
\end{aligned}
$$


with $\gamma=\frac{1}{2}(d-1)+2 \alpha$. Finally, Eq. 4.1 follows from Lemma 4.1. It remains to show that Eq. 4.2 holds, i.e.

$$
\delta(d-1)-\frac{1}{2} \gamma+\alpha>0 .
$$

Using the expression for $\gamma$, it suffices to note that $\delta(d-1)-\frac{1}{4}(d-1)>0$ if $\delta \in\left(\frac{1}{4}, \frac{1}{2}\right)$, so it is possible to choose $\left[\delta^{\prime}, \delta^{\prime \prime}\right] \subset\left(\frac{1}{4}, \frac{1}{2}\right)$.

It should be noted that Eq. 4.5 can be weakened by requiring that $F$ is regular varying at zero. However, in this case the normalising factors for the diameter and the constants involved in the formula for $\sigma_{0}$ are influenced by the slowly varying part of $F$.

Using similar arguments, it is possible to check Eqs. 2.4 and 2.5 if $\xi=\eta v$ for independent $\eta$ and $u$, where $\eta$ is distributed on $[0,1]$ and $v$ is distributed on $\mathbb{S}^{d-1}$ with a bounded density with respect to the surface area measure, see also Lao and Mayer (2007). An example of $v$ having a purely atomic distribution is considered in Section 5.5.

\section{Examples}

\subsection{Uniform Distribution on the Ball}

Consider the case of random points uniformly distributed in $B$.

Theorem 5.1 As $n \rightarrow \infty$, the diameter of the convex hull of a homogeneous Poisson process $\Pi_{\lambda}$ with intensity $\lambda=n / \mu_{d}(B)$ restricted on the d-dimensional unit ball, $d \geq 2$, has limit distribution

$$
\mathbf{P}\left\{n^{\frac{4}{d+3}}\left(2-\operatorname{diam} \Pi_{\lambda}\right) \leq t\right\} \rightarrow 1-\exp \left\{-\frac{1}{2} c t^{\frac{d+3}{2}}\right\}, \quad t>0,
$$

where

$$
c=\frac{2^{d+1} d \Gamma\left(\frac{d}{2}+1\right)}{\sqrt{\pi}(d+1)(d+3) \Gamma\left(\frac{d+1}{2}\right)} .
$$

Proof The tail behaviour of $\left\|\xi_{1}\right\|$ is determined by

$$
\mathbf{P}\left\{\left\|\xi_{1}\right\| \geq 1-s\right\}=1-\frac{\mu_{d}((1-s) B)}{\mu_{d}(B)}=1-(1-s)^{d},
$$

so that Theorem 4.2 is applicable with $\alpha=1$ and $a=d$.

By the de-Poissonisation argument, Theorem 5.1 yields Theorem 1.1. Note that in case $d=2$ the constant $c$ equals $16 /(15 \pi)$, which also corresponds to the bounds given in Eq. 1.1. The tail behaviour of $\|\tilde{\xi}\|$ can also be obtained from the explicit formula for the distribution of the length of a random chord in the unit ball, see Kendall and Moran (1963, 2.48). 
5.2 Uniform Distribution on the Sphere

Another example of a spherically symmetric distribution is given by the uniform distribution on $\mathbb{S}^{d-1}$, i.e. if

$$
\kappa(A)=\mu_{d-1}(A) / \mu_{d-1}\left(\mathbb{S}^{d-1}\right)
$$

for all measurable $A \subset \mathbb{S}^{d-1}$. The following result follows from Theorem 4.2 in case $a=F(0)=1$ and $\alpha=0$.

Theorem 5.2 If $\Pi$ is the homogeneous Poisson process on $\mathbb{S}^{d-1}$ with the total intensity $n$, then for any $d \geq 2$

$$
\lim _{n \rightarrow \infty} \mathbf{P}\left\{n^{\frac{4}{d-1}}(2-\operatorname{diam} \Pi) \leq t\right\}=1-\exp \left\{-\frac{1}{2} c t^{\frac{d-1}{2}}\right\}, \quad t>0,
$$

where

$$
c=\frac{2^{d-1} \Gamma\left(\frac{d}{2}\right)}{(d-1) \pi^{\frac{1}{2}} \Gamma\left(\frac{d-1}{2}\right)} .
$$

Alternatively, the tail behaviour of $\|\tilde{\xi}\|$ may be derived from the explicit formula for the distribution of the distance between two uniform points on the unit sphere, see Alagar (1976).

\subsection{Distribution in Spherical Sectors}

Define $\xi_{S}=\eta v_{S}$, where $\eta \in[0,1]$ and $v_{S}$ is uniformly distributed on a set $S \subset \mathbb{S}^{d-1}$ of positive surface area measure. The distribution $\xi_{S}$ can be considered as the conditional distribution of $\xi=\eta v$ given that $v \in S$, where $v$ is uniformly distributed on $\mathbb{S}^{d-1}$. Since only symmetric points of $S$ contribute to the maximum interpoint distance, define

$$
S_{0}=\{u \in S:-u \in S\}=S \cap \check{S} .
$$

Assume that $S_{0}$ coincides with the closure of its relative interior on $\mathbb{S}^{d-1}$. Writing $\tilde{\xi}=\eta^{\prime} v^{\prime}-\eta^{\prime \prime} v^{\prime \prime}$ for every $u$ from the relative interior of $S_{0}$ and i.i.d. $v^{\prime}, v^{\prime \prime}$ uniformly distributed on $\mathbb{S}^{d-1}$, we arrive at

$$
\begin{aligned}
\mathbf{P}\left\{\tilde{\xi}_{S} \in C_{s}\left(u, z_{s}\right)\right\} & =\mathbf{P}\left\{\tilde{\xi} \in C_{s}\left(u, z_{s}\right) \mid v^{\prime}, v^{\prime \prime} \in S\right\} \\
& =\mathbf{P}\left\{\tilde{\xi} \in C_{s}\left(u, z_{s}\right), v^{\prime}, v^{\prime \prime} \in S\right\} \frac{\mu_{d-1}^{2}\left(\mathbb{S}^{d-1}\right)}{\mu_{d-1}^{2}(S)} \\
& =\mathbf{P}\left\{\tilde{\xi} \in C_{s}\left(u, z_{s}\right)\right\} \frac{\mu_{d-1}^{2}\left(\mathbb{S}^{d-1}\right)}{\mu_{d-1}^{2}(S)}
\end{aligned}
$$

for all sufficiently small $s$. If Eq. 4.5 holds, then Eq. 4.6 holds with $\sigma_{0}$ replaced by $\sigma_{0} q_{S}$ with

$$
q_{S}=\frac{\mu_{d-1}\left(S_{0}\right) \mu_{d-1}\left(\mathbb{S}^{d-1}\right)}{\mu_{d-1}^{2}(S)} .
$$


Note that $q_{S}=\mu_{d-1}\left(\mathbb{S}^{d-1}\right) / \mu_{d-1}(S)$ if $S$ is centrally symmetric, i.e. $S=S_{0}$. For instance, if $\xi$ is uniformly distributed inside the spherical sector

$$
K=\{t u: u \in S, t \in[-1,1]\}
$$

with a centrally symmetric $S \subset \mathbb{S}^{d-1}$ of positive surface area measure, then Eq. 5.1 holds with $c$ replaced by $c q_{S}$.

\subsection{Non-uniform Angular Distributions}

Assume that $\xi$ is distributed on the boundary of the unit circle in $\mathbb{R}^{2}$ according to some not necessarily symmetric probability measure $\kappa$, which can be then considered a measure on $[0,2 \pi)$. If $\xi_{1}$ and $\xi_{2}$ are distributed on $[0,2 \pi)$ according to $\kappa$, then

$$
\begin{aligned}
\mathbf{P}\left\{1-\cos \left(\xi_{1}-\xi_{2}\right)\right. & \left.\leq 2 s(1-s / 2)(1-s)^{-2},\left|\xi_{1}+\xi_{2}-2 u\right| \leq 2 s^{\delta}\right\} \\
& \leq \mathbf{P}\left\{\tilde{\xi} \in C_{s}\left(u, s^{\delta}\right)\right\} \\
& \leq \mathbf{P}\left\{1-\cos \left(\xi_{1}-\xi_{2}\right) \leq 2 s,\left|\xi_{1}+\xi_{2}+\pi-2 u\right| \leq 2 s^{\delta}\right\},
\end{aligned}
$$

where the addition of angles is understood by modulus $2 \pi$. Thus, $\mathbf{P}\{\tilde{\xi} \in$ $\left.C_{s}\left(u, s^{\delta}\right)\right\}$ is equivalent as $s \downarrow 0$ to

$$
\mathbf{P}\left\{\left|\xi_{1}+\xi_{2}\right| \leq 2 \sqrt{s},\left|\xi_{1}+\xi_{2}+\pi-2 u\right| \leq 2 s^{\delta}\right\}
$$

Assume that the distribution $\kappa$ has bounded density $f$ with respect to the length measure on the unit circle. Then the probability above is equivalent to

$$
2 \sqrt{s} 4 s^{\delta} f(u) f(u+\pi)=4 s^{\gamma}\left(2 s^{\delta}\right) f(u) f(u+\pi) .
$$

Thus, Eq. 2.4 holds with $\gamma=\frac{1}{2}$ and

$$
\sigma_{0}=4 \int_{0}^{2 \pi} f(u) f(u+\pi) d u .
$$

The boundedness of $f$ also implies that Eq. 2.5 holds, so that the limit distribution is given by Eq. 2.9. In particular if $\kappa$ is uniform on the circle, then $f(u)=(2 \pi)^{-1}$, so that $\sigma_{0}=4 /(2 \pi)=2 / \pi$, so that

$$
\lim _{n \rightarrow \infty} \mathbf{P}\left\{n^{4}\left(2-\operatorname{diam} \Pi_{n \kappa}\right) \leq t\right\}=1-e^{-\frac{1}{\pi} \sqrt{t}}, \quad t \geq 0
$$

which also corresponds to the result of Theorem 5.2 for $d=2$.

\subsection{Segments and Disks in the Unit Ball}

Assume that $L_{1}, \ldots, L_{m}$ are segments that are obtained by intersection the unit ball with $m$ different lines passing through the origin. Assume that $\mathbf{P}\left\{\xi \in L_{i}\right\}=p_{i}$ for $i=1, \ldots, m$, and conditionally on $\xi \in L_{i}, \xi$ is distributed according to the length measure on $L_{i}=\left[-x_{i}, x_{i}\right]$. The one-dimensional result 
for the range of a uniform random sample (Galambos 1978, Sec. 2.9) implies that

$$
\lim _{s \downarrow 0} \mathbf{P}\left\{\tilde{\Pi}_{n \kappa}\left(C_{s}\left(x_{i}, z_{s}\right)\right) \geq 1\right\}=1-\left(1+\frac{1}{2} t p_{i}\right) e^{-\frac{1}{2} t p_{i}}=g\left(t, x_{i}\right),
$$

where $n=t / s$, i.e. Eq. 2.13 holds with $\gamma=2$ and $\sigma$ being a purely atomic measure with unit atoms at $\left\{ \pm x_{i}, i=1, \ldots, m\right\}$. Theorem 2.9 implies that

$$
\lim _{n \rightarrow \infty} \mathbf{P}\left\{n\left(2-\operatorname{diam} \Pi_{n \kappa}\right) \leq t\right\}=1-e^{-\frac{1}{2} t} \prod_{i=1}^{m}\left(1+\frac{1}{2} t p_{i}\right) .
$$

If $L_{1}, \ldots, L_{m}$ are obtained as intersections of the unit ball with linear subspaces of possibly different dimensions, then only those of these subspaces with the smallest dimension contribute to the asymptotic distribution of the maximum interpoint distance. If the smallest dimension is at least 2 , then $\sigma$ is non-atomic and Theorem 2.7 is applicable as in the case of uniformly distributed points in a lower-dimensional unit ball. Otherwise, we arrive at the above formula for segments.

Consider now a countable family of segments $L_{i}=\left[-x_{i}, x_{i}\right], i \geq 1$. Without loss of generality assume that $x_{i} \rightarrow x_{0}$ as $i \rightarrow \infty$ and $x_{i} \neq x_{0}$ for all $i$. Let $v$ be the measure on $\mathbb{S}^{d-1}$ with atoms $\pm x_{i}$ with $v\left(\left\{x_{i}\right\}\right)=v\left(\left\{-x_{i}\right\}\right)=p_{i}, i \geq 1$. In comparison with the case of a finite number of segments, we need also to find the limit of $\mathbf{P}\left\{\tilde{\Pi}_{n \kappa}\left(C_{s}\left(x_{0}, z_{s}\right)\right) \geq 1\right\}$ as $s \downarrow 0$. Notice that $v\left(C_{s}\left(x_{0}, z_{s}\right)\right)=q_{s} \rightarrow 0$ as $s \downarrow 0$. Thus, $\mathbf{P}\left\{\tilde{\Pi}_{n \kappa}\left(C_{s}\left(x_{0}, z_{s}\right)\right) \geq 1\right\}$ is bounded above by the probability that the Poisson point process with the total intensity $n q_{s}$ on $\left[-x_{0}, x_{0}\right]$ has diameter that exceeds $2-s$. Using the one-dimensional result, it is easy to see that the corresponding limit is zero if $\gamma=2$. In order to arrive at a non-trivial limit, we need to set $\gamma>2$, which is impossible, since the normalisation $n^{\gamma}$ is too big for the diameters of the Poisson processes restricted on the individual segments $L_{i}, i \geq 1$. Therefore, Eq. 5.4 holds in this case with the infinite product, i.e. for $m=\infty$.

For instance, assume that $p_{i}=6 \pi^{-2} i^{-2}, i \geq 1$, where $\zeta$ is the zeta-function. Using a formula for infinite product (Hansen 1975, 89.5.16) we obtain

$$
\lim _{n \rightarrow \infty} \mathbf{P}\left\{n\left(2-\operatorname{diam} \Pi_{n \kappa}\right) \leq t\right\}=1-e^{-\frac{1}{2} t} \frac{\sinh \sqrt{3 t}}{\sqrt{3 t}} .
$$

\subsection{Distributions Supported by Pointed Sets}

Let $\kappa$ be a probability measure supported by a closed (not necessarily convex) set $K \subset B$. Assume that $K$ has a unique diameter of length 2, i.e. $K$ contains exactly two symmetric points $u_{0}$ and $-u_{0}$ with norm 1 . Define

$$
\begin{aligned}
E_{1}(s) & =\left\{x \in K:\left\langle x, u_{0}\right\rangle \geq 1-s\right\}, \\
E_{2}(s) & =\left\{x \in K:\left\langle x,-u_{0}\right\rangle \geq 1-s\right\}, \\
q(s) & =\max \left\{\left|\operatorname{arc} u_{1}\right|+\left|\operatorname{arc} u_{2}-\pi\right|: u_{i} \in E_{i}(s), i=1,2\right\},
\end{aligned}
$$


where $\operatorname{arc} u$ denotes the angle between $u$ and $u_{0}$. Note that $E_{1}(s) \downarrow\left\{u_{0}\right\}$ and $E_{2}(s) \downarrow\left\{-u_{0}\right\}$ as $s \downarrow 0$. By $W_{a}(\beta)$ we define a random variable with the Weibull distribution given by

$$
\mathbf{P}\left\{W_{a}(\beta) \leq t\right\}=1-e^{-a t^{\beta}} .
$$

The following result generalises the main result of Appel et al. (2002) for the multidimensional case.

Theorem 5.3 Assume that

$$
\lim _{s \downarrow 0} s^{-\beta} \kappa\left(E_{i}(s)\right)=a_{i}
$$

for some $\beta_{i}, a_{i} \in(0, \infty), i=1,2$. Further assume that $s^{-\frac{1}{2}} q(s) \rightarrow 0$ as $s \downarrow 0$. If $\beta_{1} \neq \beta_{2}$, then $n^{\frac{1}{\beta}}\left(2-\right.$ diam $\left.\Pi_{n \kappa}\right)$ converges in distribution to $W_{a}(\beta)$, where $\beta$ is the smaller of $\beta_{1}, \beta_{2}$ and a equals the corresponding $a_{i}$. If $\beta_{1}=\beta_{2}=\beta$, then $n^{\frac{1}{\beta}}\left(2-\right.$ diam $\left.\Pi_{n \kappa}\right)$ converges in distribution to the sum $W_{a_{1}}(\beta)+W_{a_{2}}(\beta)$ of two independent Weibull-distributed random variables.

Proof Since $\lim _{s \downarrow 0} s^{-\frac{1}{2}} q(s)=0$, we get

$$
\mathbf{P}\left\{\tilde{\Pi}_{n \kappa}\left(C_{s}\left(u_{0}, s^{\delta}\right)\right) \geq 1\right\}=\mathbf{P}\left\{\operatorname{diam} \Pi_{n \kappa} \geq 2-s\right\},
$$

for sufficiently small $s>0$ and any $\delta<\frac{1}{2}$. The following inequalities are obvious for some $c>0$

$$
\begin{aligned}
\mathbf{P}\left\{\operatorname{diam} \Pi^{*} \geq 2-s-c q^{2}(s)\right\} & \leq \mathbf{P}\left\{\operatorname{diam} \Pi_{n \kappa} \geq 2-s\right\} \\
& \leq \mathbf{P}\left\{\operatorname{diam} \Pi^{*} \geq 2-s\right\},
\end{aligned}
$$

where

$$
\Pi^{*}=\left\{\left\langle\xi_{i}, u_{0}\right\rangle: \xi_{i} \in \Pi_{n \kappa}\right\}
$$

is the orthogonal projection of $\Pi_{n \kappa}$ onto the line passing through $-u_{0}$ and $u_{0}$. By the properties of the Poisson process and the assumptions of the theorem we get

$$
\mathbf{P}\left\{\operatorname{diam} \Pi^{*} \geq 2-s\right\}=\mathbf{P}\left\{\max \Pi^{*}-\min \Pi^{*} \geq 2-s\right\}
$$

where

$$
\begin{aligned}
\mathbf{P}\left\{\max \Pi^{*} \geq 1-s\right\} & =1-\exp \left\{-n \kappa\left(E_{1}(s)\right)\right\}, \\
\mathbf{P}\left\{-\min \Pi^{*} \geq 1-s\right\} & =1-\exp \left\{-n \kappa\left(E_{2}(s)\right)\right\} .
\end{aligned}
$$

An application of Theorem 2.9 yields the result with $t=s n^{1 / \min \left\{\beta_{1}, \beta_{2}\right\}}$.

This result could easily be extended to cover cases where the support $K$ of $\kappa$ contains countably many disjoint chords of length 2 , e.g. if $\kappa$ is the uniform measure on a hexagon. 
Acknowledgements The authors are grateful to Jürg Hüsler for comments and helpful discussions and to the referee for correcting several mistakes and misprints. This study was supported by the Swiss National Foundation Grant No. 200021-103579.

\section{References}

Alagar, V.S.: The distribution of the distance between random points. J. Appl. Probab. 13, 558-566 (1976)

Appel, M.J.B., Najim, C.A., Russo, R.P.: Limit laws for the diameter of a random point set. Adv. Appl. Probab. 34, 1-10 (2002)

Bräker H., Hsing, T.: On the area and perimeter of a random convex hull in a bounded convex set. Probab. Theory Relat. Fields 111, 517-550 (1998)

Bräker, H., Hsing, T., Bingham, N.H.: On the Hausdorff distance between a convex set and an interior random convex hull. Adv. Appl. Probab. 30, 295-316 (1998)

Dümbgen, L., Walther, G.: Rates of convergence for random approximations of convex sets. Adv. Appl. Probab. 28, 384-393 (1996)

Galambos, J.: The Asymptotic Theory of Extreme Order Statistics. Wiley, New York (1978)

Gneiting, T.: On $\alpha$-symmetric multivariate characteristic functions. J. Multivar. Anal. 64, 131-147 (1998)

Groeneboom, P.: Limit theorems for convex hulls. Probab. Theory Relat. Fields 79, 327-368 (1988)

Gruber P.M.: Aspects of approximation of convex bodies. In: Gruber, P.M., Wills, J.M. (eds.) Handbook of Convex Geometry, vol. A, B, pp. 319-345. North-Holland, Amsterdam (1993)

Hansen E.R.: A Table of Series and Products. Prentice-Hall, Englewood Cliffs, NJ (1975)

Henze N., Klein T.: The limit distribution of the largest interpoint distance from a symmetric Kotz sample. J. Multivar. Anal. 57, 228-239 (1996)

Kendall, M.G., Moran, P.A.P.: Geometrical Probability. Charles Griffin, London (1963)

Lao, W.: The Limit Law of the Maximum Distance of Points in a Sphere in $R^{d}$. Tech. rep., University of Karlsruhe, Karlsruhe (2006)

Lao, W., Mayer, M.: U-max-statistics. arXiv.org, math.ST/0704.1379 (2007)

Matthews, P.C., Rukhin, A.L.: Asymptotic distribution of the normal sample range. Ann. Appl. Probab. 3, 454-466 (1993)

Mattila, P.: Geometry of Sets and Measures in Euclidean Spaces. Cambridge University Press, Cambridge (1995)

Molchanov, I.: Theory of Random Sets. Springer, London (2005)

Molchanov, I.S.: On the convergence of random processes generated by polyhedral approximations of compact convex sets. Theory Probab. Appl. 40, 383-390 (1995)

Penrose, M.: Random Geometric Graphs. Oxford University Press, Oxford (2003)

Reitzner, M.: Central limit theorem for random polytopes. Probab. Theory Relat. Fields 133, 483-507 (2005)

Schneider, R.: Random approximations of convex sets. J. Microsc. 151, 211-227 (1988)

Schneider, R.: Convex Bodies. The Brunn-Minkowski Theory. Cambridge University Press, Cambridge (1993)

Schreiber, T.: Limit theorems for certain functionals of unions of random closed sets. Theory Probab. Appl. 47, 79-90 (2003)

Silverman, B.W., Brown, T.C.: Short distances, flat triangles and Poisson limits. J. Appl. Probab. 15, 815-825 (1978) 